\title{
Fake News, Immigration, and Opinion Polarization
}

\author{
Carlo Alessandro Borella
}

MS in Economics, Barcelona Graduate School of Economics, Barcelona, Spain

\begin{abstract}
Nowadays, it is hard to venture online without coming across a heated discussion over "Fake News"; as a result, people are finding hardE times moving through an entirely new distorted era of misinformation and biased news. In this paper, we investigate the effect of fake news on people's opinion polarization on a hot topic - such as immigration - through an experiment. We show that "Backfire Effect" and a cognitive bias we named "Validation Myopia" occur when people read Fake news independently if they believe them or not. In addition, we show how Fake news affect opinion polarization and we provide evidence that the Backfire Effect has a higher magnitude than its counterpart (i.e. validation myopia). Finally we show that the emotion-driven effect of fake news can be neutralized thanks to ex-ante signaling of the inaccuracy of fake news.
\end{abstract}

Keywords: fake news, opinion polarization, immigration, backfire effect, validation myopia, post-truth.

JEL Classification: C90.

(C) The Author, 2017. This article is published with open access at ARMG Publishing.

\section{Introduction}

Illegal immigrants live in five-stared hotels entirely paid by Italian taxpayers, Pope Francis endorsed President Donald Trump in the 2016 election and dangerous chemtrails are left in the sky by high-flying aircrafts...right? Wrong. These are just some of hundreds of fake news that have been published in the last years. Many on those stories have been shared millions of times over Facebook, Twitter and other socialmedia platforms.

Designed to supply misinformation spread via traditional print and broadcast news media or online social media - fake news have the intention to mislead in order to orientate people's opinions on politics, science and society, often with exaggerated, sensationalist, manifestly false headlines that grab the attention of the readers. Even if it might seem a new phenomenon, Fake news has spread around throughout the entire human history. For instance, significant fake news stories can be traced back to Octavian's $1^{\text {st }}$ century campaign of misinformation against Mark Antony (Soll, 2016), while in 1782, Benjamin Franklin printed a fake edition of an actual Boston newspaper, The Independent Chronicle. Among the untrue commercials and articles, Franklin inserted a made-up story about the massive massacre of white settlers on the borders of New York. According to the news, Native Americans, in the service of the British forces, had collected 700 scalps from men, women, children, and even infants (Nilsson, 2017).

Nowadays, fake news represents a serious issue that often leads to real implications. The structure of social media platforms dramatically differs from previous media technologies. From this perspective, contents can be relayed amongst users with no significant third-party filtering, fact-checking, or editorial judgment; in practice a single user with no credentials or reputation can in some cases reach as many readers as CNN, The Washington Post, or the New York Times enhancing this unrestrained spread of false news and stories.

A noticeable example is the massive amount of fake news that aimed to discredit the Italian President of the Chamber of Deputies Laura Boldrini, who has been - as a consequence - the target of rape and other threats on social-media ${ }^{1}$. Even more worrying are the potential implications that fake news had on US elections in 2016. A fake news report, that Democratic senators wanted to impose sharia in Florida, was repeated by Michael Flynn, Trump's nominee for national security adviser. (Flynn also tweeted a "Must Read" link about Democrats' "sex crimes with children"). Another false report, that Trump supporters chanting "we

\footnotetext{
${ }^{1}$ For instance, controversial claims on immigration and taxation have been often erroneously attributed to her, leading to a hate escalation on Facebook and other social media.
} 
hate Muslims, we hate blacks, we want our great country back" at a rally, was reported as true on election night $^{1}$.

Is then Fake news so difficult to detect? The short answer would be "yes, it is". A study from Stanford History Education Group (2016) suggests that people find hard times distinguishing truthful pieces of information from false ones, besides the sample analyzed was composed of all educated individuals. It is then evident that uncertainty on the reliability of information and the threats of Fake news are likely to affect somehow people's opinion formation processes. With our work we aim to shed light on the implications of Fake news in opinion formation, in particular we are interested in Fake news as a stimulus that potentially exacerbates opinion polarization.

First, we will answer the question "does fake news exacerbate opinion polarization?" Then we will show that the effect of fake news is mainly emotion-driven (in the sense that is not related to a person's perception of the credibility of a news). We also investigated on the psychological effects that lead opinion polarization. Finally we will provide evidence that the biasing effect of fake news can be neutralized if the "falsity" of the news is revealed ex-ante.

\section{Background}

As already mentioned, previous literature emphasized the difficulty encountered by people in detecting fake news. For instance, Allcott \& Gentzkow (2017), analyzing the effects of false news circulated on social media on the 2016 US elections, found out that in the three months before the election, fake news favoring Trump were shared a total of 30 million times on Facebook, while those favoring Clinton were shared 8 million times. In addition, they also showed how $14 \%$ of Americans considers social media their main source of information and they brought evidence that people are much more likely to believe stories that favor their preferred candidate, especially if they have ideologically ghettoized social media networks.

Previous research investigated also the role of sampling processes in opinion formation. Social media provides fertile ground in this sense, since people can sample news according to their tastes, and this could potentially generate polarization (people could decide to read only articles that represent their prior beliefs, cementing as a consequence their opinions). However extant empirical findings about the connection between online political communication and opinion polarization are mixed. Studies on online selective exposure (e.g. Iyengar \& Hahn, 2009) are counterbalanced by studies on accidental or unintentional exposure (e.g. Brundidge, 2010); research showing the extent of homophily in social media networks (e.g. Colleoni, Rozza, \& Adam, 2014; Levi, 2012) likewise appears alongside research showing the positive relationship between social media use and network diversity (e.g. Kim, Hsu, \& Gil de Zuniga, 2013).

Nonetheless, in our work we are not interested in the sampling processes that might affect opinion polarization (as the ones mentioned above). Our focus is the role of Fake news in opinion polarization through psychological mechanisms. We considered as main references two well-known psychological effects, namely the confirmation bias and the backfire effect: the former is the is the tendency to search for, interpret, favor, and recall information in a way that confirms one's preexisting beliefs or hypotheses (Baron, 2000); the latter occurs when, in the face of contradictory evidence, established beliefs do not change but actually get stronger.

In presence of fake news we expect people to get polarized in their opinion with respect to the topic presented. In our study we selected the topic of illegal immigration. The reason for this choice relies on the relevance and the actuality of this topic that is largely debated in many European and non-European countries. Thousands of fake news are shared on social media platforms every day, most of them depicting immigrants as a dangerous threat for occidental countries. Commonly, this kind of news pushes the idea that immigrants are mostly terrorists, they bring diseases etc., through "informing" about fictitious crime facts that in reality never happened (Juhász \& Szicherle, 2017).

As additional note, in our analysis we define as "Validation Myopia" the effect for which people exacerbate their prior beliefs on a topic independently of the credibility on the piece of information that is confirming their beliefs. Unlike the Confirmation bias, - that is related to the tendency of people to rely in the authenticity of fake news that confirm one's opinion and the biased sampling process of information that confirms one's priors - the validation myopia works with no hypothesis on the credibility of the stimulus. Precisely, it

\footnotetext{
${ }^{1}$ Hunt, E. (2016). What is fake news? How to spot it and what you can do to stop it. The Guardian, December.
} 
is a cognitive bias for which people strength their opinions through emotive-stimuli (i.e. fake news) even if the credibility of the stimulus is not perceived as truthful (but note that the stimulus is not presented as fake). This effect perfectly fits with the context of post-truth reality, the political culture in which debate is framed largely by appeals to emotion disconnected from the details of policy, and by the repeated assertion of statements to which factual confutations are ignored ${ }^{1}$.

\section{Method}

Subjects. A total of 150 participants (42\% males, aged between 19 and 72 with an average age of 31 and 21 countries of origin in total) have been involved in our experiment answering an anonymous survey on Qualtrics.

Design and methodology. In order to test our hypothesis (i.e. fake news exacerbates opinion polarization) we designed three online questionnaires. Every participant was randomly assigned to one of them. The first part of the survey was common to all the participants. It consisted in a series of statements the participants had to rate from 0 to 10 where 0 stands for "completely disagree" and 10 "completely agree". In particular we tried to identify their level of openness and nationalism and their emotional control level. The first would be a good predictor of people's opinion about immigration (the more one is open toward diversity the more she or he is likely to have a positive opinion about immigrants, the opposite works with nationalism). The latter helps us to better address the psychological effect of fake news. We expected that people with higher levels of emotional response are easily susceptible to fake news, since in many cases the content of Fake news aims to move readers' emotional response more than convince with facts.

In the second part we asked participants to read fake news about immigration in Sweden where "(..) Sexual abuse from newly arrived migrants is not only prevalent in schools but that victims are accused of being racist if they speak out." ${ }^{2}$ For treatment 1 the article was presented as a legitimate article whereas in treatment 2 we stated directly to the participants that the article was a made-up story. Control group had no article to read. In the last part of the questionnaire - common to each group - we asked participants to rate some statements about immigration both from an economic and a cultural perspective ${ }^{3}$. Moreover we asked some questions about the credibility and unbiasness of the article and the emotion response the survey-taker had after reading the news. Finally, we asked people from treatment 1 if they would have changed their answers about immigration if they knew that the article was fake. Here we present a sample of questions we used in the last part of the survey.

For our analysis, first, we constructed 3 indexes from the answers we obtained. In particular, we calculated opinion indexes that summarize the economic, cultural and overall opinions on immigration for each participant $i$ as follows:

$$
\begin{aligned}
& \text { Opinion Index (culture })_{i}=\frac{1}{V} \sum_{v=1}^{V} Q_{v i} \\
& \text { Opinion Index (economic) })_{i}=\frac{1}{J} \sum_{j=1}^{J} Z_{j i}
\end{aligned}
$$

Opinion Index $(\text { overall })_{i}=\frac{1}{V} \sum_{v=1}^{V} Q_{v i}+\frac{1}{J} \sum_{j=1}^{J} Z_{j i}$.

Where each index is the mean of the opinion expressed on the topic by individual $i$, (for positive affirmation about immigration we subtracted 10 and changed the sign).

We did the same for computing the Openness-Nationalism Index and the Emotional Index. Examples of statements about openness and emotional response are, namely, "I feel that the culture of my country is better than other cultures"; "I am not very skilled in controlling my emotions"4.

\footnotetext{
${ }^{1}$ Alcorn, G. (2014). Facts are futile in an era of post-truth politics.

${ }^{2}$ Refer to the Appendix for the entire article.

${ }^{3}$ As done before they had to rate from 0 to 10 each statement where 0 was "completely disagree" and 10 was "completely agree".

${ }^{4}$ Emotion response statements have been taken from "Abridged Social Skills Inventory" by Oldmeadow, J., Quinn, S. \& Kowert, R.

(2012) while Nationalism and Openness statements are taken from Lyons, P. A., Kenworthy, J. B., \& Popan, J. R. (2010).
} 
We present the distribution and the Kernel estimate of the overall opinion index in Figure 1.

As we can see from the chart, our sample on average is slightly favorable about immigration $(M=5.33>5$ where 5, as already mentioned, stands for indifferent). We also performed the Doornik-Harsen normalitytest and we cannot accept the null hypothesis of normality $\left(p=0.069, \chi^{2}=5.349\right)$.

Finally we computed a polarization index that we used as our main variable of interest as follows:

Polarization Index $(\text { cultural })_{i}=\sqrt{\frac{1}{V} \sum_{v=1}^{V}\left(Q_{v i}-5\right)^{2}}$

Polarization Index $(\text { economic })_{i}=\sqrt{\frac{1}{J} \sum_{j=1}^{J}\left(Z_{j i}-5\right)^{2}}$

Polarization Index $(\text { overall })_{i}=\sqrt{\frac{1}{V} \sum_{v=1}^{V}\left(Q_{v i}-5\right)^{2}+\frac{1}{J} \sum_{j=1}^{J}\left(Z_{j i}-5\right)^{2}}$.

The more a person expresses agreement or disagreement on the topic the higher the index is. As for the opinion index we considered polarization on opinion about immigration both as a whole and disaggregated (economic-related and cultural-related opinion about immigration). Each of the aggregated polarization indexes is given by the root squared mean of individual polarization indexes. In Figure 2 we present the distribution and the Kernel density of the overall polarization index, similar results has been found looking at the cultural and economic indexes.

As before, we performed the Doornik-Harsen normality-test also for this index and we cannot reject the null hypothesis $\left(p=0.9915, \chi^{2}=0.017\right)$; thus, our dependent variable is normally distributed.

Finally, we present the entire article we used for both the treatment groups and summary statistics of the participants in Appendix.

T-tests have been run to check if the randomization has been effective and to avoid facing spurious results; checking for each variable and between each group. We had four variables for which the three groups differed; we took into account this potential threat using those variables as control for our regressions ${ }^{1}$.

\section{Empirical strategy}

As preliminary analysis, we computed the mean polarization for each of the 15 questions presented in the survey about immigration for each of the three groups (control, treatment 1 and treatment 2). We mapped the polarization levels in Figure 3.

As we can see from the graph, for each question, the treatment group one experienced the higher levels of polarization, while control group and treatment two share almost the same levels of polarization. This suggests that the choice of the statements to present in the survey have been effective and worked all in the same direction.

For a more concrete analysis we use an OLS approximation to determine the effect of our treatments on the polarization index.

The specification of the general model is as follows:

$Y_{i}=\alpha+$ Treatment $_{i}+\beta X_{i}+f_{c}+\mu_{i}$

$Y_{i}$ is our dependent variable containing the three aggregated polarization indexes (overall, economic, cultural), on a scale from zero to five.

\footnotetext{
${ }^{1}$ In particular we had the percentage of people who lived in a foreign country that differs between control and treatment 1 groups (p.value $=0.08$ ); right wing share that is different in treatment 2 with respect to treatment 1 and control group $(p=0.2$ and 0.06 respectively) and for the share of Christians that differs between treatment 1 and control group $(p=0.01)$.
} 
Treatment is a dummy variable that equal 1 if the individual is treated and 0 otherwise. In our analysis we consider three different comparisons:

1. Treatment 1 vs. Control group.

2. Treatment 1 vs. Treatment 2 .

3. Treatment 2 vs. Control group.

$X_{i}$ is a matrix of control variables. Namely: age, sex, religion (Atheism, Agnosticism, Christianity, Islamism, Buddhism, others); higher education (1 for bachelor onwards, 0 otherwise) and a dummy for being right wing ( 1 if right wing, 0 otherwise).

We also considered country fixed effects $f_{c}$, in order to control for unobservable heterogeneity between countries since our sample includes people from different realities ( 21 countries in total, mostly from Italy and Peru).

\section{Results}

Tables 3, 4 and 5 show the results of our estimations. Columns 1 and 2 refer to the results using as outcome variable the overall polarization index, columns 3 and 4 show the results using the economic polarization index and, finally, columns 4 and 5 using the cultural polarization index for our different treatment specifications.

From Table 3 we can see that reading a fake news (without knowing it is fake) increases, on average, the overall polarization index and the economic polarization index by approximately 0.6 points and the cultural polarization index by 0.45 points. These results are robust and significant across specifications. Moreover, these results has a higher magnitude when we compare treatment one with treatment two (Table 4), the effect of the treatment 1 is approximately 0.7 points for the overall polarization and economic polarization index, and 0.5 for the cultural one. Again, this result is robust and significant across specifications.

We report the results of the experiment for each of the polarization indexes in Figures 4, 5, 6.

For the overall index treatment one group recorded higher levels of polarization $(M=2.82)$ than treatment two $(M=2.15), t(-3.51), p<0.01$; and control group $(M=2.25), t(-2.99), p<0.01$. While control group and treatment group two do not differ significantly from one another. Same results have been found using as dependent variable the economic and the cultural indexes.

As far as the economic polarization index is concerned, treatment one group has been found to be significantly more polarized $(M=2.77)$ than control group $(M=2.22), t(-3.81), p<0.01$; and treatment two $(M=2.09), t(-2.92), p<0.01$, while treatment two and control group are not significantly different.

As for the other two indexes, the cultural one seems to vary among the groups in the same way: the level of polarization of treatment one group $(M=2.78)$ is significantly higher that the polarization recorded for control group $(M=2.26), t(-2.87), p<0.01$; and treatment two, $(M=2.16), t(-2.54), p<0.01$. Moreover, control and treatment are not statistically different in terms of polarization.

Throughout all the specifications, we have strong evidence that fake news play a role in opinion polarization; moreover, we can see that this effect is homogeneous among two levels of the topic considered: the cultural issues of immigration and the economic ones (i.e. the potential implications of immigration on culture and economy). This can be inferred from Figure 3 and Figure 4 and comparing coefficients from Table 3. As we can see the mean level of economic and cultural polarization in treated group one are respectively 2.78 and 2.77 where the control groups have mean polarization 2.22 and 2.26.

This is particularly interesting if we consider that the article presented provided no information about economic burdens of immigration, while it was more related to cultural clash. This can be interpreted as if the fake news works mainly via emotions and not according to some rational response of the individual to the news presented. In particular, economic opinion on immigration is affected even if no economic issue was described in the article.

It follows that people against immigration exacerbate their general (economic and cultural) negative opinion on immigration no matter what is the focus of the negative stimulus toward immigration (in this case a cultural emphasis); while people that have positive opinion about immigrants, once exposed to the negative 
cultural stimulus, react exacerbating their positive beliefs both for cultural and economic aspects of immigration.

In addition, to check if these effects are heterogeneous across some groups we included interaction terms of the two treatments with gender, emotional control responses, openness index and level of education. Contrary to what we expected, none of these interactions terms were significant suggesting that there are no heterogeneous effects by these characteristics so that people react homogeneously to fake news independently of their background and cultural level.

We then tried to disentangle the two effects that occur when reading fake news, namely the backfire effect and the validation myopia. Considering people with negative opinions about immigration, we tested if the effect takes place just for the ones who believe in the news. However, we found no significance difference (performing a T-test) in the reaction to the news between people who claimed the news to be true and the ones who did not. This led us to consider the cognitive effect of validation myopia instead of the confirmation bias. It is also interesting to point out that our cognitive effect perfectly mirrors the backfire effect (while the confirmation bias relies on the assumption that people believe on the news).

As already mentioned, both the effects exacerbate opinion polarization working on opposite directions and for both it is not important if the news is perceived as credible. In particular, we wanted to see which has the stronger effect in magnitude.

In order to do so we proceeded as follows:

Using the control group sample we run the regression:

$$
\text { Opinion Index } \text { I }_{i}=\beta_{1} \text { Openess }_{i}+\beta_{2} \text { Emotion }_{i}+\beta_{3} X_{i}+\varepsilon
$$

Where the dependent variable is the opinion index for individual $i$ showed in section 3, Openess $s_{i}$ and

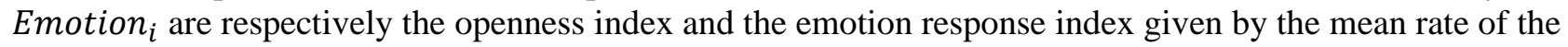
openness and emotion response questions; finally $X_{i}$ is a vector of demographic characteristics.

We present the results of the regression in Table 6, reported in Appendix. This simple model $\left(R^{2}=0.63\right)$ helps to predict peoples attitude toward immigration.

We then compute Opınıon In ex $_{\imath}$ for all the individuals in treatment 1 and control group. In this way we obtain the expected opinion on immigration for each participant ex-ante reading the fake news. We then divide the sample in two groups, depending if Opınıon Index $\lessgtr 5$, (i.e. depending if people are expected to have a positive or negative opinion about immigration). The first pro-immigration group is likely to experience the backfire effect; while the anti-immigration group is likely to experience the validation myopia.

We are using Opınıon In ex $_{\imath}$ instead of the actual opinion polarization in order to rule out possible confounding effects and endogeneity of the subdivision in these two groups due to the treatment itself. Explicitly, if we divide the treated sample depending on Opinion Index $\$$, (i.e. using the actual Opinion index) we would not consider the possibility that some people changed their mind about immigration after reading the fake news (biasing our analysis) and we would only observe something that is not an actual polarization. Thanks to this approach - that we propose starting from the LATE (Local Average Treatment Effect) concept - we can identify four groups we will use to test which effect is stronger:

$Y_{T P i}-\frac{1}{N} \sum_{i=1} Y_{C P i} \neq Y_{T N i}-\frac{1}{N} \sum_{i=1} Y_{C N i}$

Where:

$>Y_{T P i}$ is the polarization index for individual $i$, that has been treated with the news (treatment 1) and has Opınıon Index $x_{l}>5$ (i.e. has a positive opinion of immigration);

$>Y_{T N i}$ is the polarization index for individual $i$, that has been treated with the news (treatment 1) and has Opınıon Index $\leq 5$ (i.e. has a negative opinion of immigration);

$>\frac{1}{N} \sum_{i=1} Y_{C P i}$ is the mean polarization of people who have expected positive opinion about immigration and have not been treated; 
$>\frac{1}{N} \sum_{i=1} Y_{C P i}$ is the mean polarization of people who have expected negative opinion about immigration and have not been treated.

In practice, the first term capture the change in polarization of people who have negative opinion of immigrants with respect to the counterfactual mean polarization level of people who are not treated and have a negative opinion of immigrants, while the second terms works in the same way for people who have a positive opinion about immigration.

We then perform a one-sided T-test in order to see if the response to the article (in terms of polarization) of one group differs from the other (i.e. the left-hand side differs from the right-hand side) and it turned out that the people that have positive opinion about immigration are significantly more affected $(p=0.0214)$.

We display the results in Figure 4 were it is evident that the increase in polarization for people with a favorable opinion about immigrants is higher that the increase in polarization recorded for the group of people with a negative opinion ( $M=0.11$ for the first and $M=0.86$ for the second).

This provides evidence that the magnitude of the backfire is bigger than the magnitude of the validation myopia effect; suggesting that the fake news exacerbate opinion polarization in both sides (pro and cons), in particular, the induced polarization is asymmetric, since people that perceived the fake news as a threat are more affected (backfire effect is stronger). For confirming our findings we repeated the same passages using the actual Opinion Index $x_{i}$ for both control and treatment groups and we obtained the same results.

Finally, we see no significant effect when we compare treatment 2 with the control group (Table 5) in any of the polarization indexes. This suggests that showing to people that a news is explicitly false neutralize the effect of fake news on opinion polarization. This could be explained as follows: once people who have positive opinions about immigration are exposed to a stimulus that goes against their beliefs, the backfire effect is triggered and they tend to strengthen their belief as a consequence. But if we show that the stimulus is "innocuous" people would not feel it as a threat and they do not tend to emotionally read to contrast it. Similarly, if people who have negative opinion about immigration are exposed to a stimulus that supports their ideas they tend to reinforce their beliefs due to validation myopia. However, once the news is called to be false people tend to be more prudent with their emotions and keep their opinions unchanged, without exacerbating them.

\section{Limitations}

Firstly, since the respondents of the survey were not selected randomly we can face problems with the external validity of our results. For example, our sample underrepresents right-winged people (only $12 \%$ of the respondents identify themselves as rightists where $19 \%$ were centrists). In addition, 102 persons did not finish the questionnaire which could introduce endogeneity in our results because of the possible deterministic nature of this behavior (self-selection and correlation between non-finishing the survey and some of our outcomes).

However, using the partial information from unfinished surveys we run a regression (logistic model) to test if individual characteristics are correlated with the dummy variable finishing or not finishing the survey. And we found that none of the characteristics affected significantly the probability of finishing the survey. Secondly, we could face a problem of measurement error of our outcome variables and covariates. Since most respondents were Italian or Spanish speakers, but the survey questions were in English, it is possible that some respondents did not fully understand the sense of some questions and their answers are not aligned with the sense of the questions. Moreover, since it was a long questionnaire it is possible that the last questions were not carefully read including an additional source of measurement error.

\section{Conclusions}

We observe that exposure to fake news increases polarization. This increase holds when we compare Treatment 1 vs. Treatment 2 . However, contrary to what we expected we do not find heterogeneous effects across some characteristics like emotional control index, gender, openness index, etc. In particular, it seems that the perceived credibility of news is not a key factor for polarization; for this reason we considered in our analysis the cognitive bias of validation myopia that could be considered in a certain sense as a mirrored backfire effect. 
We also showed that fake news work thorough emotions more than rationality: people react through emotions without evaluating the news content and updating their opinion according to the new information sampled and its credibility). This is clear from the homogeneous effect for both economic and cultural opinion and - to some extent - from the news itself that deals with facts happening in a foreign country for which people should be more prudent in expressing opinions.

We also showed that the polarizing effect works asymmetrically in the sense that people who perceive the fake news as a threat for their beliefs get more polarized than the ones for which the fake news supports their beliefs (i.e. backfire effect is stronger than validation myopia).

Furthermore, considering the fact that there is no significant effect when we compare treatment 2 against treatment 1 , we infer that providing tools to identify fake news could drastically reduce extreme polarization and can help to reduce unproductive flame discussions on controversial topics. This finding has interesting implications: first because people are somehow conscious that fake news are likely to affect them (67\% of our sample answered that they would have expressed a different opinion about some of the statements if they knew that the news was fake) but if the "pretentious validity" of the news is not explicitly called out the polarization take place no matter the perceived credibility of the news.

Further research could be done using more sophisticated indexes for computing the emotional-control level of individuals. Moreover it could be interesting capture trace political orientation of respondents through a political chart questionnaire; while at the moment we are only asking directly for political orientation. Finally, we are taking into account the effect of fake news of only one controversial topic (immigration). Further research could investigate the psychological effects that would occur with other news that focus on another topics like abortion, the war on drugs, politics, etc., addressing the validation myopia with further experiments. It would be also interesting to analyze how the magnitude of polarization changes depending on the level of sensationalism the fake news presented.

\section{References}

1. Allcott, H. and Gentzkow, M. (2017). Social Media and Fake news in the 2016 Election. Journal of Economic Perspectives, 31(2), 211-236.

2. Baron, J. (1995). Myside bias in thinking about abortion. Thinking \& Reasoning, 1(3), 221-235.

3. Baron, J. (2012). Thinking and deciding. Cambridge: Cambridge University Press.

4. Brundidge, J. (2010). Encountering "Difference" in the Contemporary Public Sphere: The Contribution of the Internet to the Heterogeneity of Political Discussion Networks. Journal of Communication, 60(4), 680-700.

5. Colleoni, E., Rozza, A. and Arvidsson, A. (2014). Echo Chamber or Public Sphere? Predicting Political Orientation and Measuring Political Homophily in Twitter Using Big Data. Journal of Communication, 64(2), 317-332.

6. Iyengar, S. and Hahn, K. (2009). Red Media, Blue Media: Evidence of Ideological Selectivity in Media Use. Journal of Communication, 59(1), 19-39.

7. Kim, Y., Hsu, S. and de Zúñiga, H. (2013). Influence of Social Media Use on Discussion Network Heterogeneity and Civic Engagement: The Moderating Role of Personality Traits. Journal of Communication, 63(3), 498-516.

8. Lyons, P. A., Kenworthy, J. B., \& Popan, J. R. (2010). Ingroup identification and group-level narcissism as predictors of U.S. citizens' attitudes and behavior toward Arab immigrants. Personality and Social Psychology Bulletin, 36(9), 1267-1280.

9. Nilsson, J. (2017). Ben Franklin Used Fake News.

10. Siebörger, R. (2017). Fake News, Alternative Facts, History Education. Public History Weekly, 8.

11. Soll, J. (2016). The Long and Brutal History of Fake News.

12. Stanford History Education Group (2015). Evaluation Information: The Cornerstone of Civil Online Reasoning. 


\section{Appendices}

Table 1. Examples of statements about immigration

\section{Cultural Statements}

Illegal Immigrants do not share the values from my country and they do not want to integrate.

Sometimes illegal Immigrants want to influence religious beliefs from my country.

Economic Statements

Illegal immigrants do provide quality, non-skilled labor which benefits our country

Children of illegal immigrants are an economic burden and harm our welfare system.

Note: We used 15 statements in total, where 6 were about economy, 5 about culture and the rest could apply to both.

Table 2. Summary statistics

\begin{tabular}{|l|c|c|c|}
\hline & $C$ & $T_{1}$ & $T_{2}$ \\
\hline Male \% & 0.38 & 0.39 & 0.50 \\
\hline Left wing \% & 0.45 & 0.57 & 0.42 \\
\hline Centrist \% & 0.14 & 0.20 & 0.21 \\
\hline Right \% & 0.07 & 0.09 & 31 \\
\hline Age mean & 31 & 29 & 0.67 \\
\hline Lived in a foreign country \% & 0.57 & 0.74 & 0.38 \\
\hline "I value much religion" \% & 0.39 & 0.43 & 4.70 \\
\hline Emotional index mean & 4.58 & 4.01 & 0.79 \\
\hline Higher education \% & 0.71 & 0.83 & 6.38 \\
\hline $\begin{array}{l}\text { "Immigration is an issue" } \\
\text { mean rate }\end{array}$ & 6.87 & 6.64 & 5.22 \\
\hline Openness index mean \% & 5.26 & 5.56 & 0.44 \\
\hline Atheism/Agnosticism \% & 0.32 & 0.37 & 0.46 \\
\hline Christianism \% & 0.59 & 0.35 & $\mathbf{4 8}$ \\
\hline Total participants & $\mathbf{5 6}$ & $\mathbf{4 6}$ & \\
\hline
\end{tabular}

Note: $C=$ Control group, $T_{1}=$ treatment $1, T_{2}=$ treatment 2 .

Table 3. Treatment 1 vs. Control

\begin{tabular}{|c|c|c|c|c|c|c|}
\hline Variables & $\begin{array}{c}(1) \\
\text { Overall }\end{array}$ & $\begin{array}{c}(2) \\
\text { Overall }\end{array}$ & $\begin{array}{c}(3) \\
\text { Economic }\end{array}$ & $\begin{array}{c}(4) \\
\text { Economic }\end{array}$ & $\begin{array}{c}\text { (5) } \\
\text { Cultural }\end{array}$ & $\begin{array}{c}\text { (6) } \\
\text { Cultural }\end{array}$ \\
\hline Treatment & $\begin{array}{c}0.562^{* * * *} \\
(0.188)\end{array}$ & $\begin{array}{l}0.550^{* * *} \\
(0.219)\end{array}$ & $\begin{array}{c}0.549^{* * * *} \\
(0.188)\end{array}$ & $\begin{array}{c}0.565^{* * * *} \\
(0.211)\end{array}$ & $\begin{array}{l}0.516^{* * *} \\
(0.203)\end{array}$ & $\begin{array}{l}0.415^{*} \\
(0.243)\end{array}$ \\
\hline Constant & $\begin{array}{c}2.253^{* * *} \\
(0.126)\end{array}$ & $\begin{array}{c}2.296^{* * *} \\
(0.330)\end{array}$ & $\begin{array}{c}2.222^{* * * *} \\
(0.126)\end{array}$ & $\begin{array}{l}2.300^{* * * *} \\
(0.319)\end{array}$ & $\begin{array}{c}2.262^{\text {**** }} \\
(0.136)\end{array}$ & $\begin{array}{c}2.514^{* * * *} \\
(0.367)\end{array}$ \\
\hline Observations & 102 & 102 & 102 & 102 & 102 & 102 \\
\hline$R^{2}$ & 0.082 & 0.307 & 0.079 & 0.353 & 0.061 & 0.252 \\
\hline Covariates & $\mathrm{NO}$ & YES & NO & YES & $\mathrm{NO}$ & YES \\
\hline Country FE & $\mathrm{NO}$ & YES & $\mathrm{NO}$ & YES & $\mathrm{NO}$ & YES \\
\hline
\end{tabular}

Note: Standard errors in parentheses ${ }^{* * *} p<0.01,{ }^{* *} p<0.05,{ }^{*} p<0.1$.

\footnotetext{
${ }^{1}$ We took off from the sample the observations for the countries in which immigration is not a hot topic.
} 
Table 4. Treatment 1 vs. Treatment 2

\begin{tabular}{|c|c|c|c|c|c|c|}
\hline Variables & $\begin{array}{c}(1) \\
\text { Overall }\end{array}$ & $\begin{array}{c}(2) \\
\text { Overall }\end{array}$ & $\begin{array}{c}(3) \\
\text { Economic }\end{array}$ & $\begin{array}{c}\text { (4) } \\
\text { Economic }\end{array}$ & $\begin{array}{c}\text { (5) } \\
\text { Cultural }\end{array}$ & $\begin{array}{c}(6) \\
\text { Cultural }\end{array}$ \\
\hline Treatment 3 & $\begin{array}{c}0.667^{* * * *} \\
(0.190)\end{array}$ & $\begin{array}{l}0.591^{* *} \\
(0.225)\end{array}$ & $\begin{array}{c}0.685^{\text {***** }} \\
(0.180)\end{array}$ & $\begin{array}{c}0.652^{* * * *} \\
(0.205)\end{array}$ & $\begin{array}{c}0.622^{\text {**** }} \\
(0.217)\end{array}$ & $\begin{array}{l}0.470^{*} \\
(0.265)\end{array}$ \\
\hline Constant & $\begin{array}{c}2.253^{* * * *} \\
(0.133)\end{array}$ & $\begin{array}{l}1.818^{* * * *} \\
(0.373)\end{array}$ & $\begin{array}{c}2.086^{* * * *} \\
(0.126)\end{array}$ & $\begin{array}{l}1.692^{* * * *} \\
(0.340)\end{array}$ & $\begin{array}{c}2.156^{* * * *} \\
(0.152)\end{array}$ & $\begin{array}{c}2.060^{* * * *} \\
(0.439)\end{array}$ \\
\hline Observations & 94 & 94 & 94 & 94 & 94 & 94 \\
\hline$R^{2}$ & 0.118 & 0.324 & 0.136 & 0.385 & 0.082 & 0.252 \\
\hline Covariates & $\mathrm{NO}$ & YES & $\mathrm{NO}$ & YES & $\mathrm{NO}$ & YES \\
\hline Country FE & $\mathrm{NO}$ & YES & $\mathrm{NO}$ & YES & $\mathrm{NO}$ & YES \\
\hline
\end{tabular}

Note: Standard errors in parentheses ${ }^{* * *} p<0.01,{ }^{* *} p<0.05,{ }^{*} p<0.1$.

Table 5. Treatment 2 vs. Control

\begin{tabular}{|l|c|c|c|c|c|c|}
\hline \multicolumn{1}{|c|}{ Variables } & $\begin{array}{c}(1) \\
\text { Overall }\end{array}$ & $\begin{array}{c}(2) \\
\text { Overall }\end{array}$ & $\begin{array}{c}(3) \\
\text { Economic }\end{array}$ & $\begin{array}{c}(4) \\
\text { Economic }\end{array}$ & $\begin{array}{c}(5) \\
\text { Cultural }\end{array}$ & $\begin{array}{c}(6) \\
\text { Cultural }\end{array}$ \\
\hline \multirow{2}{*}{ Treatment 2} & -0.105 & -0.133 & -0.136 & -0.162 & -0.106 & -0.110 \\
& $(0.190)$ & $(0.202)$ & $(0.189)$ & $(0.197)$ & $(0.199)$ & $(0.218)$ \\
\hline \multirow{2}{*}{ Constant } & $2.253^{* * *}$ & $2.047^{* * *}$ & $2.222^{* * *}$ & $2.009^{* * *}$ & $2.262^{* * *}$ & $2.242^{* * *}$ \\
& $(0.129)$ & $(0.304)$ & $(0.129)$ & $(0.296)$ & $10.135)$ & 104 \\
\hline Observations & 104 & 104 & 104 & 104 & 104 & 104 \\
\hline$R^{2}$ & 0.003 & 0.344 & 0.005 & 0.376 & 0.003 & 0.305 \\
\hline Covariates & NO & YES & NO & YES & NO & YES \\
\hline Country FE & NO & YES & NO & YES & NO & YES \\
\hline
\end{tabular}

Note: Standard errors in parentheses ${ }^{* * *} p<0.01,{ }^{* *} p<0.05,{ }^{*} p<0.1$.

Table 6. Opinion index prediction

\begin{tabular}{|c|c|}
\hline Variables & $\begin{array}{c}(1) \\
\text { Immigration openness index }\end{array}$ \\
\hline Male & $\begin{array}{c}-0.028 \\
(0.401)\end{array}$ \\
\hline Agnosticism & $\begin{array}{l}-1.056 \\
(0.746)\end{array}$ \\
\hline Christianism & $\begin{array}{c}-1.443^{* * *} \\
(0.524)\end{array}$ \\
\hline Buddhism & $\begin{array}{l}-0.318 \\
(1.367)\end{array}$ \\
\hline Other religion & $\begin{array}{l}-0.274 \\
(0.768)\end{array}$ \\
\hline Left & $\begin{array}{l}1.593^{* * *} \\
(0.472)\end{array}$ \\
\hline Right & $\begin{array}{c}0.558 \\
(1.079)\end{array}$ \\
\hline Centrist & $\begin{array}{c}0.222 \\
(0.655)\end{array}$ \\
\hline Higher & $\begin{array}{c}0.496 \\
(0.607)\end{array}$ \\
\hline Openness index mean & $\begin{array}{l}-0.059 \\
(0.166)\end{array}$ \\
\hline Emotional index mean & $\begin{array}{l}-0.017 \\
(0.124)\end{array}$ \\
\hline
\end{tabular}


Table 6 (cont.). Opinion index prediction

\begin{tabular}{|l|c|}
\hline \multicolumn{1}{|c|}{ Variables } & $\begin{array}{c}(1) \\
\text { Immigration openness index }\end{array}$ \\
\hline Lived in foreign country & -0.147 \\
$(0.500)$ & $6.148^{* * * *}$ \\
\hline Constant & $(1.310)$ \\
\hline Observations & 69 \\
\hline$R^{2}$ & 0.633 \\
\hline Covariates & YES \\
\hline Country FE & YES \\
\hline
\end{tabular}

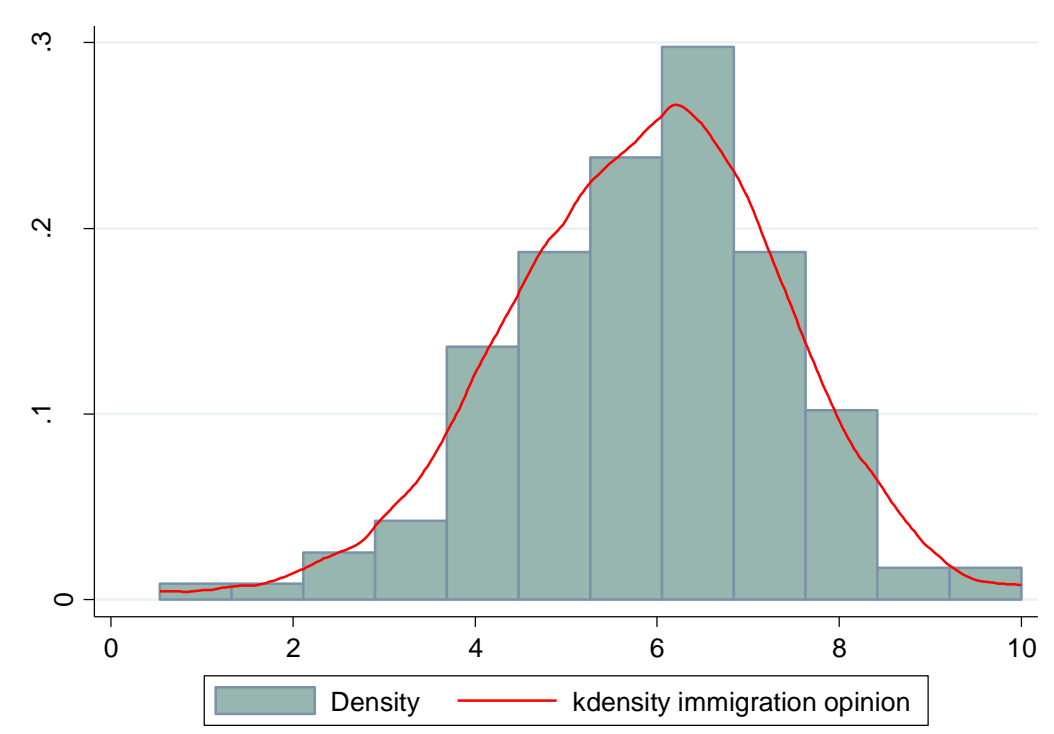

Figure 1. Density and Kernel-density (in red) of Immigration opinion index ranging from 0 to 10, for the entire sample

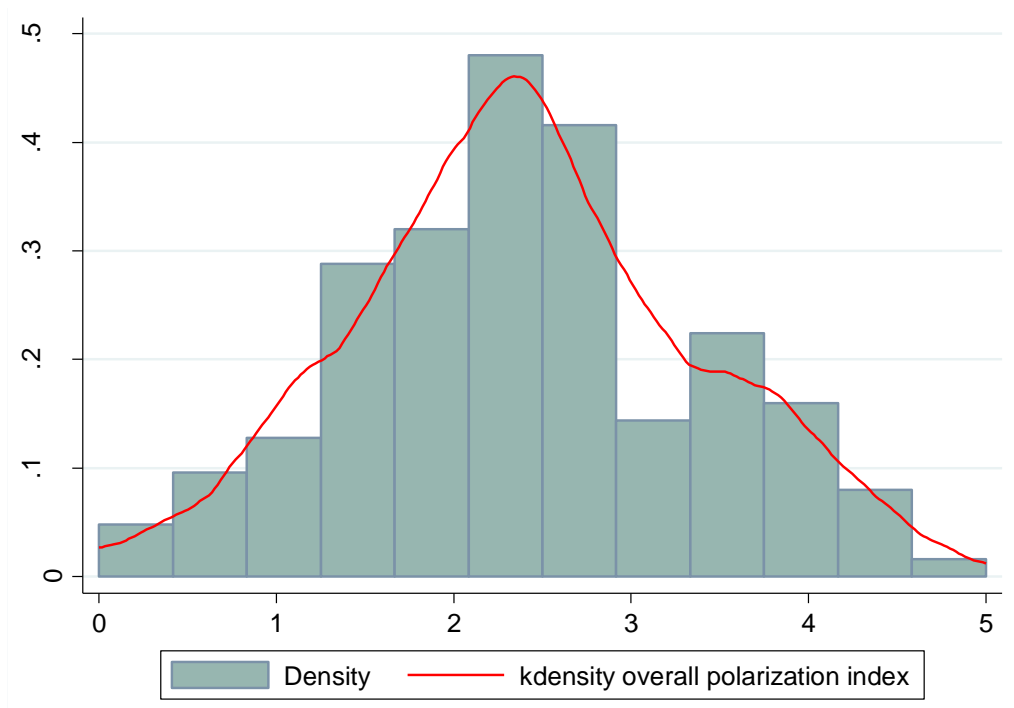

Figure 2. Density and Kernel-density of polarization index ranging from 0 to 5 , for the entire sample. 


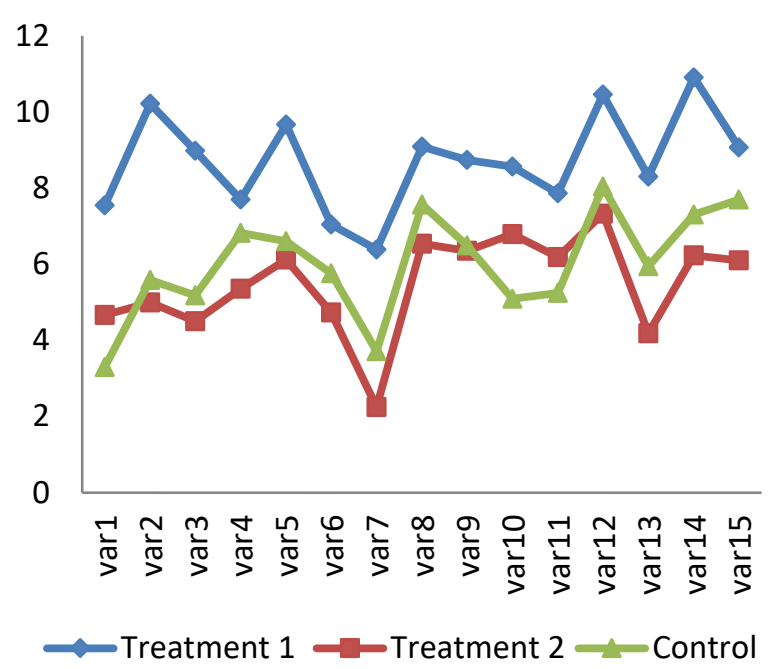

Figure 3. Mean polarization for each immigration statement. On the horizontal axis we have the questions labeled from 1 to 15 ; on the vertical axis we have the level of polarization

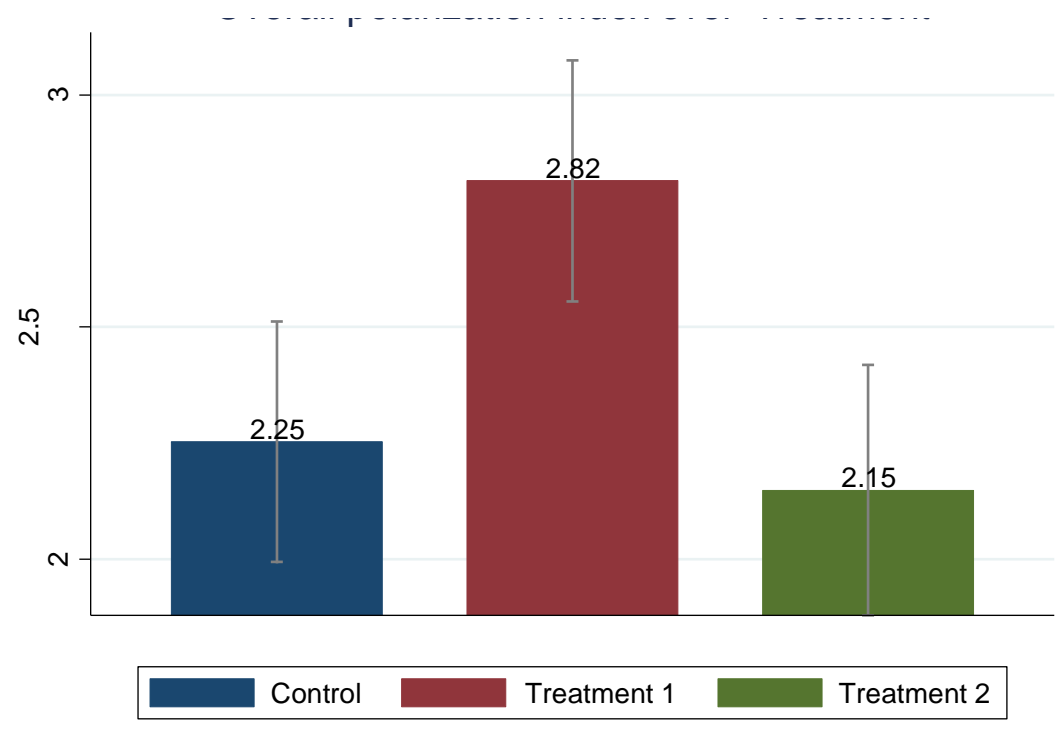

Figure 4. Results for the overall polarization index

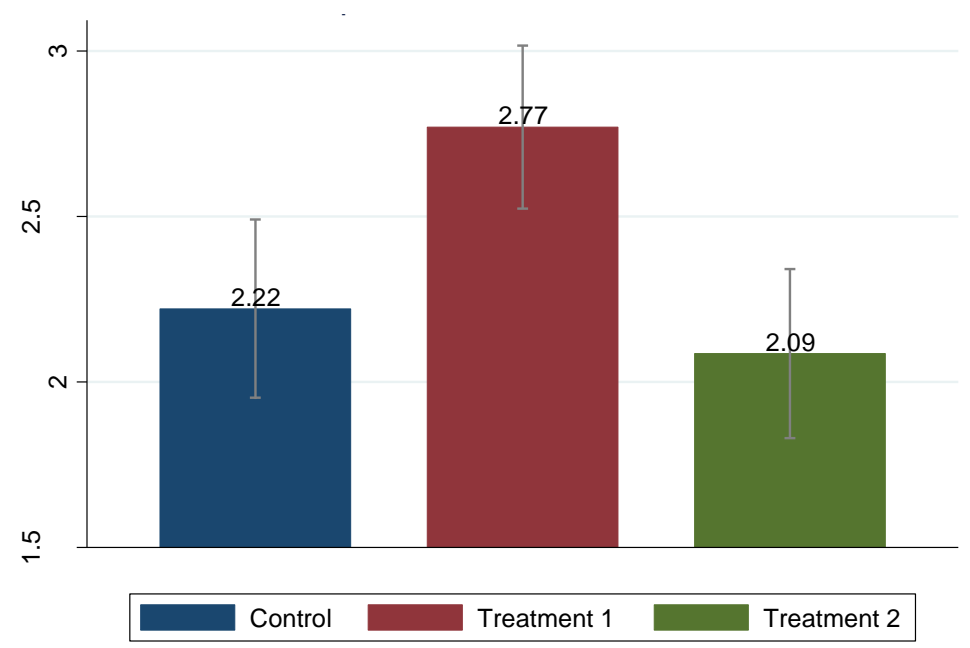

Figure 5. Results for the economic polarization index 


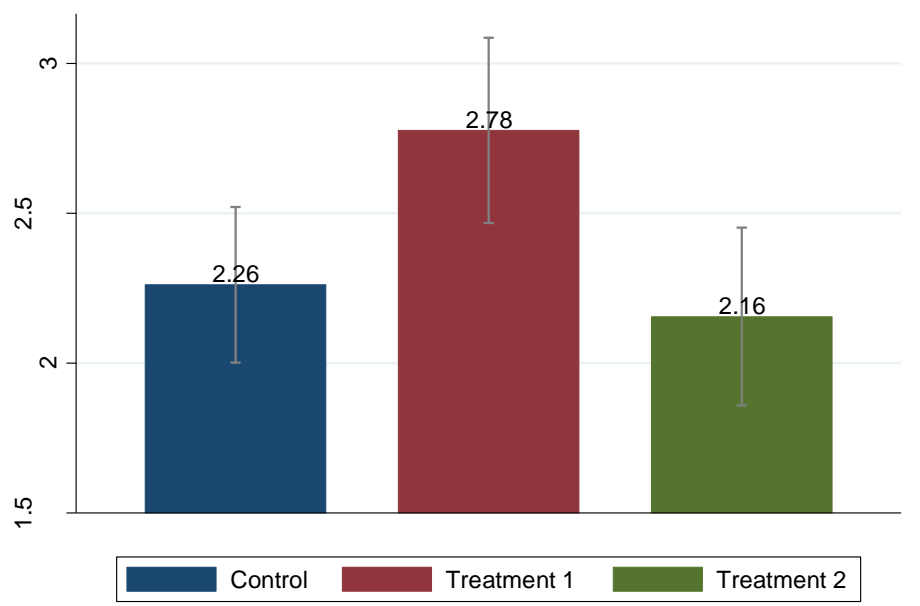

Figure 6. Results for the cultural polarization index

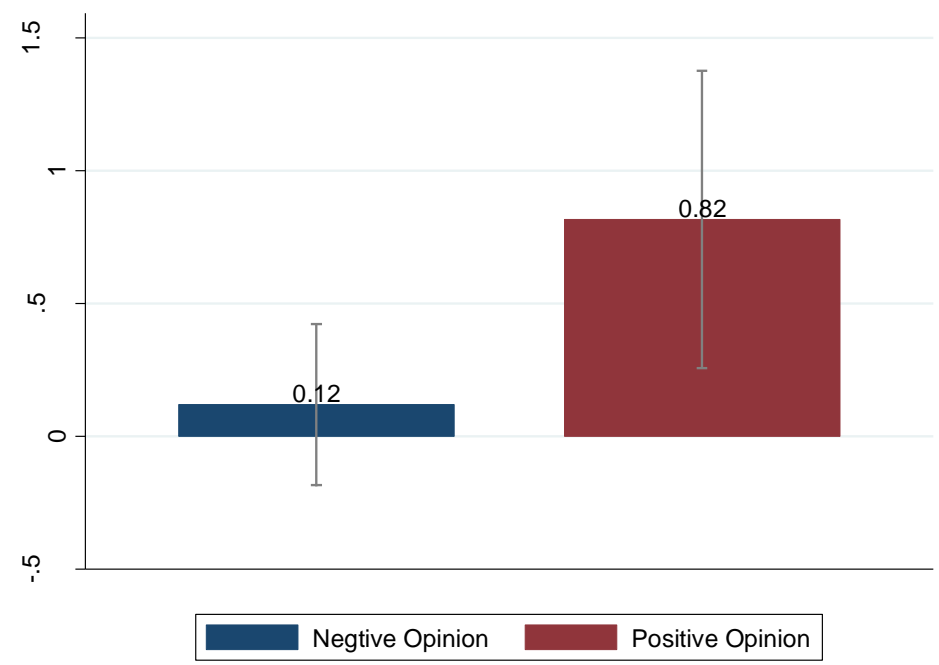

Figure 7. Comparison of the variation in polarization with respect to the control group for pro-immigration $t$ cons-immigration individuals 
Table 7. The fake news presented in treatment 1 and 2

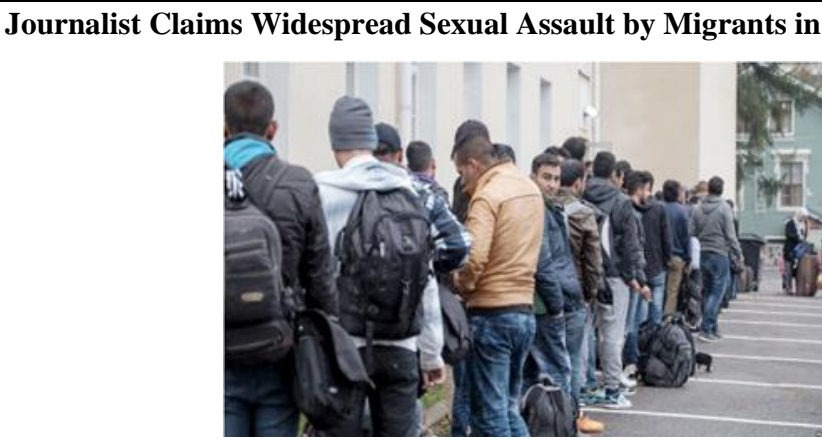

Independent Swedish journalist Joakim Lamotte has claimed sexual abuse from newly arrived migrants is not only prevalent in schools but that victims are accused of being racist if they speak out.

Mr. Lamotte, who formerly worked as a journalist at Sweden's state broadcaster SVT, has posted a video on his Facebook page describing a wave of sexual assaults committed by "newcomer" asylum seekers. He said when students or teachers attempt to talk about the problem they are called racists, islamophobes and nothing is done.

"Many of these guys are not even kids!" said Lamotte, who travels to Swedish schools to talk to students about sexual abuse. Sweden has had a problem with illegal migrants claiming to be underage, to receive preferential treatment from the government or more lenient sentences after committing crimes, but who have turned out to be adults.

A recent report showed that 23 out of 50 recent migrants from Morrocco who had claimed to be underage were actually adults. The Swedish government has been working with countries like Morrocco to use fingerprint data to properly identify many illegal migrants who arrive without any identification or with false papers.

Lamotte spoke about one school he had visited where migrant sex abuse was prevalent saying: "Young girls tell me they are sexually harassed by newcomer guys both at school and outside of school. They say they do not feel safe in the school corridors. They say they have stopped going home by themselves after school. They say they do not take the bus either." "They are called racists and islamophobes when they talk about their reality and what they face" from the staff at the schools, he said. Having visited schools every week for over a year and a half, Lamotte said he has seen the same story play out again and again.

The journalist notes the problem is not just limited to large cities but is also taking place in much smaller communities. "This is about seeing reality as it is and have the courage to talk about it, otherwise we cannot do anything about it," he said.

Source: CNN-trending.com.

Note: for treatment 2 " $100 \%$ fake news" written in red, was displayed above the article. 